\title{
On the harmonic boundary of an open Riemann surface, I
}

\author{
By Yukio KusunoKI and Shin'ichi MoRI \\ (Received August 31, 1959)
}

Every open Riemann surface $R$ can be represented as a dense subset of a compact Hausdorff space $R^{*}$ considered by Gelfand and Royden (cf. [3], [4], [10]). The ideal boundary of $R$ is then realized as a closed non-dense subset $\Gamma$ of $R^{*}$, which is, by definition, the set of maximal ideals containing the ideal $K$ of $B D$ functions with compact carriers. The set $\Delta$ of maximal ideals containing $\bar{K}$ (closure of $K$ by $B D$-topology) constitutes an essential part of $\Gamma$ and is called the harmonic boundary of $R$.

In this article we shall study some properties on harmonic boundaries and noncompact subregions on $R$. By a non-compact subregion $G$ on $R$ we shall understand in the sequel a non-compact domain on $R$ whose relative boundary $\partial G$ consists of an at most countable number of disjoint analytic Jordan curves not accumulating to any point of $R$.

1. Lemma 1. (Mori-Ota [8]) Every HBD-function on $R$ attains its maximum and minimum on $\Delta$.

LeMma 2. Let $M$ be a point of $\Delta=\Delta(R)$ and $E$ be a closed subset of $\Delta$ which is non-empty and disjoint with $M$. Then there exists a function $u_{M} \in H B D$ on $R$ such that $u_{M}(M)=1$ and $u_{M}=0$ on $E$.

Proof. From the topology (cf. [4]) of $R^{*}$ we see that there is a function $u \in B D$ which belongs to the ideal $\cap_{N \in E} N$, but not to $M$. Let $u=u_{0}+\varphi$ be the orthogonal decomposition such that $u_{0}=$ proj. $u$ (projection of $u$ to $H B D$-space) $\in H B D$ and $\varphi \in \bar{K}$ (Royden [9]). Since $\varphi=0$ on $\Delta, u_{0}(M)=u(M) \neq 0$ and $u_{0}(N)=u(N)$ $=0$ for any point $N \in E$. Hence $u_{M}=u_{0} / u_{0}(M)$ fulfils our conditions, q. e. d.

Lemma 3. Suppose that $\Delta_{1}$ and $\Delta_{2}$ be two non-empty closed subsets of $\Delta$ such that $\Delta_{1} \cup \Delta_{2}=\Delta$ and they are mutually disjoint. Then there exists a (non-negative) function $u \in H B D$ on $R$ such that $u=1$ on $\Delta_{1}$ and $u=0$ on $\Delta_{2}$.

Proof. For each point $M \in \Delta_{1}$, we consider the above function $u_{M} \in H B D$ on $R$ such that $u_{M}(M)=1$ and $u_{M}=0$ on $\Delta_{2}$, and associate a neighborhood $\left\{p ; u_{M}(p)>1 / 2\right\}$. Then, from the compactness of $\Delta_{1}$, it can be covered by a finite number of such neighborhoods $\left\{p: u_{M_{i}}(p)>1 / 2\right\} \quad\left(M_{i} \in \Delta_{1}, i=1,2, \cdots, k\right)$. Then $v_{1}=\operatorname{proj} .\left(\sum_{i=1}^{k} u_{M_{i}}{ }^{2}\right)$ $\in H B D$ vanishes on $\Delta_{2}$ while $v_{1}>1 / 4$ on $\Delta_{1}$. Analogously there exists a function $v_{2} \in H B D$ such that $v_{2}=0$ on $\Delta_{1}$ and $v_{2}>1 / 4$ on $\Delta_{2}$. Thus an $H B D$-function $v=v_{1}+v_{2}$ is greater than $1 / 4$ throughout $R^{*}$ by Lemma 1 . Hence there exists a $B D$-function $1 / v$ on $R$. The projection $u$ of $B D$-function $v_{1} / v$ to $H B D$-space obviously fulfils our conditions, which completes the proof.

Let $G$ be a non-compact subregion on $R$ and $\hat{G}$ denotes the double of $G$ with 
respect to $\partial G$. Then we note that if $\varphi \in \bar{K}=\bar{K}(R)$, the symmetric extension $\hat{\varphi}$ of $\varphi$ on $\hat{G}$ belongs to $\bar{K}(\hat{G})$. Indeed, let $\left\{\varphi_{n}\right\}$ be a sequence of elements of $K(R)$ such that $\varphi_{n} \rightarrow \varphi$ (in $B D$ ) on $R$. Then $\hat{\varphi}_{n} \in K(\hat{G})$, that is, the carriers are compact in $\hat{G}$ and the Dirichlet integrals $D_{\hat{G}}\left[\hat{\varphi}_{n}\right]$ over $\hat{G}$ are finite. Obviously $D_{\hat{G}}[\hat{\varphi}-\hat{\varphi}]$ $\leqq 2 D_{R}\left[\varphi-\varphi_{n}\right]$. Thus $\hat{\varphi}_{n}$ are uniformly bounded and $\hat{\varphi}_{n} \rightarrow \hat{\varphi}$ (in $B D$ ) on $\hat{G}$, which imply $\hat{\varphi} \in \bar{K}(\hat{G})$.

2. In the following we shall denote by $\sigma(R)$ the number of harmonic boundary points of a Riemann surface $R$. Obviously $\sigma(R)=0$ if and only if $\bar{K}=B D$, i. e. $1 \in \bar{K}$. It is known ([9]) that $1 \in \bar{K}$ is equivalent to $R \in O_{G}$. But we shall give now another simple proof. First if the harmonic measure of $\Gamma$ is zero, we know immediately that $1 \in \bar{K}$. Conversely, if $1 \in \bar{K}$, any $u \in H B D$ on $R$ belongs to $\bar{K}$; hence there is a sequence $\left\{\varphi_{n}\right\}$ such that $\varphi_{n} \in K, \varphi_{n} \rightarrow u$ (in $B D$ ) on $R$. Since $\left|D_{R}\left(u, u-\varphi_{n}\right)\right|^{2} \leqq D_{R}(u) D_{R}\left(u-\varphi_{n}\right) \rightarrow 0$ for $n \rightarrow \infty$, it follows that $D_{R}(u)=\lim _{n \rightarrow \infty} D_{R}\left(u, \varphi_{n}\right)$ $=0$ by Green's formula, hence $u \equiv$ const. Now consider the harmonic measure $\omega$ $=\omega\left(p, \Gamma, R-\bar{R}_{0}\right)$ on $R-\bar{R}_{0}\left(R_{0}\right.$ is a compact domain $)$ and its anti-symmetric extension $\hat{\omega}$ on the double $\left(R-\bar{R}_{0}\right)^{\wedge}$, then $\hat{\omega}$ is an $H B D$-function on $\left(R-\bar{R}_{0}\right)^{\wedge}$. Since the symmetric extension 1 of $1 \in \bar{K}$ belongs to $\bar{K}(\hat{G}), \hat{\omega}$ must reduce to a constant, i. e. $\omega \equiv$ const, which proves our assertion. From the proof we see also that if $G$. is a non-compact subregion on $R \in O_{G}$, then $\hat{G}$ belongs to $O_{G}$.

Proposition 1. Let $G$ be a non-compact subregion on an open Riemann surface $R$. If the double $\hat{G}$ of $G$ with respect to $\partial G$ is of hyperbolic type, the $\bar{G}$ (closure of $G$ in $R^{*}$ ) contains harmonic boundary points of $R$.

Proof. Since $\hat{G} \notin O_{G}, \sigma(\hat{G})>0$ and hence for any non-negative $\hat{\phi} \in \bar{K}(\hat{G})$ we have $\inf _{G} \hat{\phi}=0$, in particular by a remark in sec: 1

$$
\inf _{G} \varphi=0 \text { for any non-negative } \varphi \in \bar{K}=\bar{K}(R) .
$$

This shows also that the original surface $R$ is of hyperbolic type. Now suppose that $\bar{G} \cap \Gamma$ have no points of $\Delta$, then for each point $M \in \bar{G} \cap \Gamma$ we can construct a non-negative function $f_{M} \in \bar{K}$ on $R$ such that $f_{M}(M)>0$. For example, take a function $g_{M} \in \bar{K}$ which does not belong to the maximal ideal $M$, then $g_{M}{ }^{2}$ would fulfil the conditions. Since $\bar{G} \cap \Gamma$ is compact, by means of above functions $f_{M}$ we can easily get a function $\phi \in \bar{K}$ such that $\phi \geqq 0$ on $\bar{G} \cap \Gamma$. Next consider a Green function $g(p, q)$ of $R$ and

$$
g_{r}(p)=\min [g(p, q), r] \in \bar{K}
$$

where $r$ is a positive number such that the level curve $g=r$ is compact. Then $\varphi=g_{r}+\phi \in \bar{K}$ is continuous and positive on $\bar{G}$, which contradicts (*), q. e. d.

COROLlaRY (Kuramochi [5]). Let $R$ be a hyperbolic Riemann surface and $g(p, q)$ be its Green function, then the double of domain $G_{\lambda}=\{p ; g(p, q)>\lambda>0\}$ with respect to $\partial G_{\lambda}$ is a parabolic Riemann surface, unless it is compact.

Proposition 2. Let $G$ be a non-compact subregion on an open Rieman surface $R$ such that $G \notin S O_{H D}$, that is, there exists a non-constant HD-function on $G$ which 
vanishes on $\partial G$. Then $\bar{G}-\overline{\partial G}\left(R^{*}\right)$ contains some harmonic boundary points of $R$.

Proof. First we note that the double $\hat{G}$ of $G$ does not belong to $O_{H D} \supset O_{G}$, hence $\bar{K}$ contains harmonic boundary points $\Delta_{1}$ of $R$. According to A. Mori [7] there exists a non-constant $H B D$-function $u_{0}$ on $G$ which vanishes on $\partial G$. Without loss of generality we may assume that $M=\sup _{G} u_{0}>0$. Then $F=\left\{p ; u_{0}(p)>M-\varepsilon>0\right\}$ is a non-compact subregion on $G$ and we find that $\bar{F}$ contains also harmonic boundary points $\Delta_{2}\left(\subset \Delta_{1}\right)$ of $R$ and that $\Delta_{2} \subset \bar{G}-\overline{\partial G}$.

PROPOSITION 3.1.

$$
\begin{aligned}
& \sigma(\mathrm{R})=0 \text { if and only if } R \in O_{G}, \\
& \sigma(R)=1 \text { if and only if } R \in O_{H D}-O_{G} .
\end{aligned}
$$

It remains to prove the latter. Suppose that $R \notin O_{H D}$, i. e. there is a nonconstant $H D$-function $u$ on $R$, then $G_{\lambda}=\left\{p ; u(p)>\lambda, \inf _{R} u<\lambda<\sup _{R} u\right\}$ and $G_{\lambda}{ }^{c}=\{p ; u(p)<\lambda\}$ are disjoint non-compact subregions on $R$. Since $G_{\lambda}$ and $G_{\lambda}{ }^{c}$ do not belong to $S O_{H D}, \sigma(R) \geqq 2$ by Prop. 2. Thus if $\sigma(R)=1$, we see that $R \in O_{H D}-O_{G} . \quad$ Next if $\sigma(R) \geqq 2$, there would exist a non-constant $H B D$-function of $R$ by Lemma 2, which proves the converse.

3. Let $O_{H D_{n}}(n=1,2, \cdots)$ be the classes of Riemann surfaces whose ideal boundaries consist of at most $n$ maximal $H D$-indivisible sets (ConstantinescuCornea [2]). Then we have

Proposition 3.2. The following three statements are equivalent:

(i) $R \in O_{H D_{n}}$; $\quad$ (ii) $\sigma(R) \leqq n$;

(iii) The dimension of $H B D$-space on $R$ is $\leqq n$.

Hence we have $\sigma(R)=n$ if and only if $R \in O_{H D_{n}}-O_{H D_{n-1}}(n \geqq 2)$.

Since we can readily see from [2] the relation between (i) and (iii), we shall here note the equivalence of (ii) and (iii) only. Suppose $\sigma(R) \geqq n+1$ then for $n+1$ points $\left\{M_{i}\right\}$ of $\Delta$ we would have, by Lemma $2, n+1$ linearly independent $H B D$ functions $u_{i}$ on $R$ such that $u_{i}\left(M_{i}\right)=1$ and $u_{i}\left(M_{j}\right)=0(i \neq j)$. To see the converse, take above functions $u_{1}, u_{2}, \cdots, u_{m}(m=\sigma(R) \leqq n)$. Suppose that there are at least $n+1(\geqq 2)$ linearly independent functions $\omega_{i} \in H B D$, then $\omega_{i}-\sum_{j=1}^{m} \omega_{i}\left(M_{j}\right) u_{j}(i=1,2, \cdots$, $n+1)$ vanish on $\Delta$, hence these are reduced to zero identically on $R$ by Lemma 1 . Thus $\omega_{i}$ would become linearly dependent, which is absurd.

Proposition 4. If $1 \leqq \sigma(R)<+\infty$, then the genus of $R$ is infinite.

Proof. Let $\left\{R_{n}\right\}_{n=0,1}, \cdots$ be an exhaustion of $R$. Since $R$ is of hyperbolic type by Prop. 3.1, the harmonic measure $\omega=\omega\left(p, \Gamma, R-\bar{R}_{0}\right)$ is not identically zero. Suppose the genus of $R$ is finite, then for large $n, R-R_{n}$ become of planar character and a subregion $G$ such as $\left\{p ; \omega(p)>m, m=\sup _{R_{n}-R_{0}} \omega<1\right\}$ lies in $R-R_{n}$. Since a non-constant function $\omega-m \in H B D$ vanishes on $\partial G, G$ (on $R \in O_{H D_{n}}-O_{G}$ ) must belong to $O_{A D}$ (Kuramochi [6]). This is absurd, because there exists a function which maps $G$ conformally onto a plane region with finite area, q. e. d.

As an extension of Bader-Parreau's theorem [1] $(m=2)$ we have the following

Proposition 5. Let $G_{1}, G_{2}, \cdots, G_{m}$ be non-compact subregions on $R$ which are disjoint each other. If there exist non-constant $H D$-functions $U_{i}$ on $G_{i}+\partial G_{i}$ which 
vanish on $\partial G_{i}(i=1,2, \cdots, m)$, then $\sigma(R) \geqq m$, i.e. $R \notin O_{H D m-1}$. The converse is also true.

Proof. Since each $G_{i} \notin S O_{H D}$, by Prop. $2 \bar{G}_{i}-\partial \bar{G}_{i}\left(R^{*}\right)$ contains at least one point of $\Delta$, hence $\sigma(R) \geqq m$. Conversely, let $M_{1}, M_{2}, \cdots, M_{m}$ be $m$ points of $\Delta$, then since these points are mutually disjoint by the topology of $R^{*}$ (cf. [4]), there are $m$ functions $u_{i} \in H B D$ on $R$ such that $u_{i}\left(M_{i}\right)=1$ and $u_{i}\left(M_{j}\right)=0(i \neq j)$. Hence we find easily that, for suitable constants $\lambda_{i}, m$ domains $G_{i}{ }^{*}=\left\{u_{i}>\lambda_{i}\right\}$ become disjoint on $R^{*}-\Gamma$. It suffices now to transform $G_{i}{ }^{*}$ into $R$ and set $U_{i}=u_{i}-\lambda_{i}$.

4. Proposition 6. Let $G$ be a non-compact subregion of $R$ such that $G \in N O_{H D}$, that is, there does not exist any non-constant $H D$-functions on $G$ with vanishing normal derivatives on $\partial G$. If the double $\hat{G}$ of $G$ with respect to $\partial G$ does not belong to $O_{H D}$, then $\sigma(\hat{G})=2$.

Proof. First of all, we note that any function of $H B D$ on $\hat{G}$ is decomposed into a constant and an anti-symmetric function. In fact, for any $u \in H B D$ on $\hat{G}$ we write

$$
\begin{gathered}
u=u_{1}+u_{2}, \\
u_{1}(p)=(u(p)+u(\tilde{p})) / 2, \quad u_{2}(p)=(u(p)-u(\tilde{p})) / 2,
\end{gathered}
$$

where $\tilde{p}$ denotes the symmetric point of $p$ with respect to $\partial G$. Since the normal derivative of $u_{1}$ vanishes on $\partial G, u_{1}$ must be a constant. In the following we denote by $u_{0}$ the anti-symmetric part of an $H B D$-function $u$ on $\hat{G}$. Now since $\hat{G} \notin O_{H D}$ $=O_{H B D}$, there exists a non-constant function $u \in H B D$ on $\hat{G}$. Consider the orthogonal decomposition of $u_{0}^{2}$ (丰 const) such that

$$
u_{0}^{2}=v+\varphi=v_{0}+k+\varphi
$$

where $v \in H B D(\hat{G}), \varphi \in \bar{K}(\hat{G})$ and $k$ is a constant. It follows $\varphi=-k$ on $\partial G$. We note that $k$ is positive. In fact, consider an exhaustion $\left\{R_{n}\right\}$ of $R$ and harmonic functions $v_{n}$ on $\left(R_{n} \cap G\right)^{\wedge}$ with boundary values $u_{0}^{2}$, then $v_{n}-u_{0}^{2} \geqq 0$ on $\left(R_{n} \cap G\right)$ since $u_{0}{ }^{2}$ is subharmonic. It follows that $v \geqq u_{0}^{2}$ for $n \rightarrow \infty$, where the equality never holds. Otherwise $v \equiv u_{0}^{2}$ by minimum principle, that is, $u_{0}^{2}$ becomes harmonic. This is possible only if $u_{0} \equiv$ const. Now let $\tilde{\varphi}$ be a $B D$-function on $\hat{G}$ such that $\tilde{\varphi}=\varphi^{2}$ on $G$ and $\tilde{\varphi}=k^{2}$ on $\tilde{G}=\hat{G}-G$. Then $\tilde{\varphi}=0$ on $\bar{G} \cap \Delta(\hat{G})$, while $\tilde{\varphi}=k^{2}(\neq 0)$ on $\overline{\bar{G}} \cap \Delta(\hat{G})$. Thus we find that $\overline{\partial G}$ does not contain any point of $\Delta(\hat{G})$, that is, $\Delta(\hat{G})$ is split into two compact sets $\Delta_{1}(\subset \bar{G} \cap \Delta(\hat{G}))$ and $\Delta_{2}(\subset \overline{\widetilde{G}} \cap \Delta(\hat{G}))$, which are nonempty on account of $\hat{G} \notin O_{G}$. Thus we know by Lemma 3 that there exists a function $w \in H B D$ on $\hat{G}$ such that $w=1$ on $\Delta_{1}$ and $=0$ on $\Delta_{2}$. Next for any function $U \in H B D$ on $\hat{G}$ consider the orthogonal decomposition of $U w$ :

$$
U w=h+\phi=h_{0}+\gamma+\phi
$$

where $h \in H B D(\hat{G}), \phi \in \bar{K}(\hat{G})$ and $\gamma$ is a constant. From the property of $w$ it follows that $h=0$ on $\Delta_{2}$ and $h=U$ on $\Delta_{1}$. Hence

$$
h_{0}=-\gamma \text { on } \Delta_{2} \text { and } h_{0}=-\gamma+U \text { on } \Delta_{1} \text {. }
$$

Since $h_{0}$ is anti-symmetric, we find that $h_{0}=\gamma$ on $\Delta_{1}$, hence $U$ must be a constant $(2 \gamma)$ on $\Delta_{1}$. In fact, suppose $h_{0} \neq \gamma$ on $\Delta_{1}$, then there is a non-compact subregion 
$F=\left\{p ;\left|h_{0}(p)-\gamma\right|>\delta_{0}>0\right\}$ such that $\bar{F}$ contains some points of $\Delta_{1}$. It is easily seen that the symmetric region $\tilde{F}$ of $F$ with respect to $\partial G$ contains also some points of $\Delta_{2}$. While, in $\tilde{F},\left|h_{0}(\tilde{p})+\gamma\right|=\left|-h_{0}(p)+\gamma\right|>\delta_{0}$, which is absurd. Thus we know similarly that $U$ is constant on $\Delta_{2}$. From this we can conclude that $\Delta_{1}$ and $\Delta_{2}$ consists of a single point respectively, q. e.d.

From the above proof we have immediately a sufficient condition in order that $R \in O_{H D}$ (cf. [2], Satz 11).

Proposition 7. Let $G$ be a non-compact subregion on $R$. If $G \in N O_{H D}$ and $\hat{G} \notin O_{H D}$ (e.g., $G \notin S O_{H D}$ ), then $R \in U_{H D}$, i.e., there exists a non-constant $\underline{H D}$ (or $H D)$ minimal function on $R$.

It is noted that the hypothesis in Prop. 7 is not void, i. e., there exists a noncompact subregion $G$ such that $G \in N O_{H D}$ and $\notin S O_{H D}$. For example, $G=R-\bar{K}$ on $R \in O_{H D}-O_{G}$ fulfils the conditions, where $K$ is a compact domain on $R$ with analytic boundaries.

Kyoto University, and Ritsumeikan University.

\section{References}

[1] Bader, R., and M. Parreau, Domaines non compacts et classification des surfaces de Riemann. C. R. Acad. Sci. Paris 231 (1951), 138-139.

[2] Constantinescu, C., and A. Cornea, Über den idealen Rand und einige seiner Anwendungen bei Klassifikation der Riemannschen Flächen. Nagoya Math. J. 13 (1958), 169233.

[3] Gelfand, I., Normierte Ringə. Rec. Math. 9 (1941), 3-24.

[4] Gelfand, I., und G. Silow, Über varschiedene Mathoden der Einführung der Topologie in die Menge der maximalen Ideale eines normierten Ringes. Ibid. 9 (1941), 25-38.

[5] Kuramochi, Z., Harmonic measures and capacity of sets of the ideal boundary, II. Proc. Japan Acad. 31 (1955), 25-30.

[6] Kuramochi, Z., On the ideal boundary of abstract Riemann surfaces. Osaka Math. J. 10 (1958), 83-102.

[7] Mori, A., On the existence of harmonic functions on a Riemann surface. J. Fac. Sci., Univ. Tokyo 6 (1951), 247-257.

[8] Mori, S., and M. Ota, A remark on the ideal boundary of a Riemann surface. Proc. Japan Acad. 32 (1956), 409-411.

[9] Royden, H. L., Harmonic functions on an open Riemann surface. Trans. Amer. Math. Soc. 73 (1953), 40-94.

[10] Royden, H. L., On the ideal boundary of a Riemann surface. Ann. Math. Studies 30 (1953), 107-109. 